\title{
TWIN PLEAS: PROBING CONTENT AND COMPOSITIONALITY
}

\author{
Josefa Toribio \\ Department of Philosophy \\ Washington University in St. Louis
}

e-mail pepa@twinearth.wustl.edu

Philosophy and Phenomenological Research, 57, (4), 1997: 871-889.

\begin{abstract}
:
Dual factor theories of meaning are fatally flawed in at least two ways. First, their very duality constitutes a problem: the two dimensions of meaning (reference and conceptual role) cannot be treated as totally orthogonal without compromising the intuition that much of our linguistic and non linguistic behavior is based on the cognizer's interaction with the world. Second, Conceptual Role Semantics is not adequate for explaining a crucial feature of linguistic representation, viz., the special kind of compositionality known as concatenative compositionality. Dual factor theories, I conclude, cannot constitute an acceptable philosophical model of content.
\end{abstract}




\section{Introduction. A (very short) Fregean excursion.}

So called dual factor theories are proposed in an attempt to provide an explanation of the meaning of our utterances and the content of our mental states in terms that involve two different theories, each one serving separate concerns. One type of theory deals with the causal explanatory aspect of contentful mental states and/or sentences. The other type deals with those contentful mental states and/or sentences as related to propositions, i.e., as objects that can be assigned referential truth-conditions (Cfr. McGinn, 1982; Block, 1986).

I take dual component approaches to meaning to be yet another variety of the Fregean tradition. This may seem odd since Frege -but certainly not two-factor theorists- wants a thoroughly non-psychologized account of meaning. So, an explanation of my statement is needed. Because this story is well known, I will be brief.

The sense of a sentence is the thought it expresses, where this thought is understood as something objective and fully independent of our ideas in any psychological sense. Its reference is nothing but a truth-value -true or false- (Cfr. Frege, 1892). The concept of truth that arises out of postulating this kingdom of objective thoughts is, Frege believes, the necessary mediating tool between the symbols and the entities of which the world is made. The very possibility of a proper scientific account of the world can be guaranteed only by appealing to objective scientific laws that, like objective thoughts, are immune to relativistic challenges. But, at the same time, for this objective kingdom of thoughts to play the role of a mediating entity between language and world, it is necessary to introduce an essential, but new idea. The idea is that not only can we grasp those thoughts, but, also in this grasping process, their objective content -their sense- remains the same.

There is thus a double characterization of the notion of sense. On the one hand, the sense of a sentence is its truth-conditions, and to that extent, it is conceived as that which fixes the reference, that which fixes the truth or falsity of the sentence. On the other hand, the sense of a sentence is that which has cognitive value; it is that which carries some kind of information. Frege stresses the first reading of the notion of sense. The way in which the sense of a sentence determines its reference is understood as a specification of the realist truth-conditions of the sentence. But then, Frege faces the problem of explaining how it is possible for the speaker to grasp those truth-conditions, i.e., once objectivity is presupposed in that way, he faces the problem of explaining, from the cognitive point of view, how that grasping process is possible (Cfr. Dummett, 1981). 
Dual factor theorists maintain the Fregean sense / reference distinction. They stress, however, the second, cognitively oriented, characterization of sense and claim that this cognitive component must be developed within the framework of some version of so called conceptual role semantics (see below). Those same theorists claim that the referential truth-conditions of the sentences are constituted and determined independently, just in virtue of how the speaker is embedded in the world. Sense does not determine reference. Both components of meaning are -and must be-completely independent. So, the first characterization of the Fregean sense is discarded as false (Cfr. McGinn, 1982)1.

In rejecting any conceptual bridge between the two theories, dual factor advocates face a different version of the very same Fregean problem, namely, the problem of explaining how it is possible for the world to become knowable and the cognitive subjects' thoughts to become world-engaged. Although dual factor theorists insist on the distinction between the two dimensions of meaning (as though their explanatory independence were itself the key to the success of their position), I contend that, unless we want to end with the same old metaphysical problem, an account relating both theories is needed. The first part of my paper is concerned with that issue and in it, I focus on the theses defended by one of the most representative dual factor proponents, Colin McGinn. My aim is to cast doubt on the plausibility of the theoretical division proposed.

In the second part of the paper, I deal with Conceptual Role Semantics, the theory most recommended to account for one of the two dimensions of content: its causal explanatory role. I concentrate on the version of that theory proposed by Ned Block (Cfr. Block, 1986). The question I address in that part of the paper is whether or not Conceptual Role Semantics lives up to Block's claim that it is an acceptable semantics for Psychology. My question is formulated in terms of one of the conditions that, according to Block, the theory ought to meet to be satisfactory, the condition that the theory must explain compositionality. I argue that, although Conceptual Role Semantics can be used to explain a certain kind of compositionality, i.e., functional compositionality, it cannot be used to explain the kind of (concatenative) compositionality involved in linguistic representations. By showing the incapability of Conceptual Role Semantics to solve the compositionality problem, I support my contention about the impotency of dual factor theories.

1 Some advocates of dual factor theories believe that an additional account of how the two components of meaning are related is possible (Cfr. Block, 1986). I argue in Section 2.2. that the characteristics of the two factors involved preclude such an account. 


\section{The garden of the forking paths.}

The first claim I defend is that dual factor theories fail to provide an adequate account of content because in them the two factors involved in the notion of content cognitive role and referential truth conditions- are independent. To make a strong case for that contention, I show (1) why a plausible theory of content for Psychology must reconcile the cognitive and the referential dimension of content and (2) why any fully independent characterization of the two dimensions of content precludes an additional account of how the two factors are related. As I remarked, I focus mainly on Colin McGinn's proposal as representative of dual factor theories.

According to McGinn, the notion of semantic structure "falls into two" and, thus, we have "two sub-projects: we shall want to know how the truth-conditions of a sentence depend upon the reference of its parts, and we shall want to know how the cognitive role of a sentence is determined from the cognitive role of its parts" (McGinn, 1982, p. 231). In detail, McGinn's position is that the theory of reference takes the form of a Tarskian theory of truth and the theory of content takes the form of some version of conceptual role semantics, e.g., Field's probabilistic semantics. Since -as McGinn rightly points out- the epistemic concept of subjective probability and the nonepistemic concept of truth cannot be reconciled, the probabilistic and the truth-conditional semantics are depicted as fortunately independent.

For clarity, I begin by noting where I agree and where I disagree with McGinn: (i) I agree that belief-content and (sentence) meaning are hybrids, from a conceptual point of view: they are causally explanatory states of behavior and they relate to conditions in the world; (ii) I agree that "belief involves internal representations" (McGinn, 1982, p. 211) and that those representations have two aspects. However (iii) I disagree that "these two aspects must be mutually independent" (McGinn, 1982, p. The stress is mine). Here is why I think this is not the case.

There is both good and bad news concerning any kind of sense / reference distinction in the project of developing a theory of content. The good news is that the distinction captures the gap between cognitive subject and the world and thus reflects both the cognitive and the referential significance of representations. The bad news is that the metaphysical assumption on which the distinction is built cannot be explained without turning the whole project into a circular one. I now elaborate this point a bit further. 
The distinction at the semantic level works only because it is based on a symmetric metaphysics: Dual factor advocates have to presuppose a ready-made world whose existence is not dependent on the existence of beings capable of grasping the objects and properties of that world. They also have to presuppose a ready-made mind, one that can apprehend thoughts in such a way that the process of grasping them does not alter their objective content.

Given the psychologized characterization of the notion of sense present in dual factor theories and the fact that those theories have been advertised as satisfactory theories for Psychology, it is reasonable to suppose that they can provide an additional account of how the sense / reference distinction has been generated, i.e., an adequate explanation of how the split between cognition and world has come about. To fulfill that explanatory purpose, however, the theory must show how the environment surrounding the cognitive subject becomes conceptualized as an objective entity through some process of maturation of the subject's cognitive abilities, i.e., it must provide an account of learning (the acquisition of first and new concepts) and, in general, of the development of cognition. The problem is that such an explanation requires that one give up the pre-established distinction between how that world really is and how it is presented to the cognizer, the very metaphysical assumption that constitutes the core of these theories.

Now, the question is, of course, why do we need such an account? Basically because we want our theory of content to be able to sustain the plausible conviction that much of our linguistic and non-linguistic behavior is based on the cognizer's interaction with the world. We want to be able to use our theory of content to explain how it is possible for the world to become knowable and the cognitive subjects' thoughts to become world-engaged. That is especially the case when the theory is presented as an adequate theory for Psychology.

The idea underlying this plausible conviction is that cognition is not just a process of taking in information. Taking in information is possible only in the context of effective procedures that are part of the overall goal of successful negotiation of a domain. Every representation of our world implies both a dimension that is dependent on our contingent abilities to entertain a point of view towards that world -via the environment in which we are embedded- and a dimension that points at the world independently of this embodied specific point of view. If we want a theory of content that can be used to explain why systems, whose behavior involves linguistic representations, can be successful in their physical and social environments, we need a theory of content that has the conceptual resources to encompass the psychological 
aspects of content and their information-based origins. We need a theory that -unlike dual factor theories- can provide an account of how the sense / reference distinction is generated and therefore how the theories of sense and reference are related to each other.

If this is the case, and given McGinn's picture of the fortunate independence of sense and reference, it should be clear that dual factor theories fail to provide an adequate account of content because they involve the false assumption that the two factors are independent of one another. I now provide another argument to support my claim (Section 2.1). I also (Section 2.2) show that McGinn's independence claim does preclude an additional account of how the two factors are related. Block's contention that "conceptual role determines the function from context to reference and truth value" (Block, 1986, p. 644) is thereby shown as highly questionable.

2.1.

On the one hand, McGinn holds that "that which is grasped by someone who knows the meaning of a sentence is the same as that which is believed by someone whose belief is specified by using that sentence" (McGinn, 1982, p. 217). That which is believed by someone is then nothing but a proposition. It is precisely this identification that offers McGinn the prospect of transferring issues and conclusions from the area of belief to the area of meaning and it thus represents a central point in his account. On the other hand, he admits that the independence of the two factors involved in his theory of content yields the uncomfortable result that propositions do not exist, i.e., that "there is no one thing that is both the bearer of truth-value and that which determines the cognitive import of a sentence" (McGinn, 1982, p. 257, footnote 28).

On a first reading, at least, a perspective on belief-content that both incorporates as a mainstay and rules out the very notion of proposition is highly suspicious. If the reason why propositions are precluded from that theory of content is the independence of the cognitive and the referential factor, that independence should surely be questioned $^{2}$.

But this reading might be too hasty. McGinn's point is presumably that whatever determines the cognitive import of a sentence is more finely individuated than are

2 Of course, we may choose instead some Lewis-style position in relation to our conceptualizations of belief, i.e., we may think of beliefs as related not to propositions but to properties that the agent ascribes to himself or herself (Cfr. Lewis, 1979). In that case, the fact that McGinn's independence thesis precludes propositions does not seem so problematic. This, however, is not McGinn's position. 
propositions ${ }^{3}$. The fact that dual factor theories provide an independent account of those two parameters is then seen as an advantage. In this case, the independence claim allows us to account consistently for both the epistemic status of identity statements containing names and their modal status: "We can then [after having given up the assumption that sense determines reference] assign the epistemic status to the cognitive role component and the modal status to the referential component -and neither component determines the other" (McGinn, 1982, p. 246).

Even if this is the correct reading, the question still remains as to how cognitive import and the referential component relate to one another. The need for such an account is again easy to see when we place dual factor theories in their proper psychological context. Our psychological understanding of a subject's behavior depends crucially on coming to appreciate how the fruits of individual learning can affect the subject's fitness function in ways that feed back into a long-term developmental process. Yet, without a theoretical bridge connecting the cognitive import of the subject's internal states and processes and some real-world referential component, there is just no way we can account for the psychological relevance of those internal states considered as cognitive states reaching out to the world. To make psychological sense of the inner roots of the behavior of complex systems, we positively must treat their internal states as having semantic dimensions that connect with the world in a highly interactive fashion. We must, in other words, provide an explanation of how the referential properties that constitute the world out there become part of the cognizer's internal states and how the cognizer's internal states and properties help him or her to construct the world that lies out there.

Regardless of the details of any approach to content, the following seems to be right: we need truth -at the level of the theory of reference- to explain why the behaviors prompted by the understanding of conceptual roles -or stereotypes, or nonconceptual contents or whatever- are generally successful. Therefore, we need a theory of content that can be used to give an account of how these referential properties of our beliefs -the world in which the system is embedded- become the subject of the content and how that content determines those referential properties, i.e., how sense becomes world-engaged ${ }^{4}$.

3 Thanks to an anonymous referee for pointing this out to me.

$4 \quad$ McGinn himself seems to agree with this point when he says: "It is [understanding] that establishes a bridge of sorts between the theory of cognitive role and the theory of reference" (McGinn, 1982, p. 238). But the point is quickly obscured by the fact that he considers understanding to be just another propositional attitude. 


\section{2.}

Even if McGinn's claim that cognitive role and referential truth conditions should be independently characterized by a theory of meaning cannot possible be sustained, does that claim preclude an additional account of how the two factors are related? If Block is right, that should not be the case because, according to him, conceptual role could determine a function from context to reference and truth value (Cfr., Block, 1986, p. 644).

Now, as I argue in the second part of this paper, conceptual role semantics lacks the resources to meet one of the most important conditions for a theory of meaning, namely, that the theory can be used to explain concatenative compositionality (see below). If this is correct, how could the undefined notion of conceptual role determine a function from context to reference? Unlike cognitive role, reference and truth value are not holistic, context sensitive notions. The conditions that are relevant at the cognitive role level include any kind of circumstance or state of information that justifies the assertion of a given sentence. These conditions are also, in general, defeasible and include a fuzzy set of intentions, desires, and beliefs. This implies that specific circumstances warrant the assertion of a statement in some cases and not in others, depending on the interplay of such other factors. As Crispin Wright says:

the dominant problem is ... that ... justified assertion is a holistically conditioned notion. One and the same state of affairs may sometimes justify an assertion, and sometimes not, according to how other relevant background beliefs are varied. And so it looks as though the sought-for axioms would have to include, in each case, an exhaustive description of all possible relevant background beliefs together with an account of how their inclusion, or exclusion, from the state of information of the subject would affect his warrant for claiming, in specific circumstances, that a given individual (or sequence of individuals) satisfied the predicate in question (Wright, C., 1986, p. 39).

The explanatorily central notion at the cognitive level is an epistemic notion of truth and thus it is difficult to see how to construct a function from that epistemic realm to the nonepistemic realms of reference and truth value. The key point may be to realize that Block's claim is not that conceptual role determines reference but that "the 
conceptual role factor determines the nature of the referential factor" (Block, 1986, p. 644. The stress is mine). Such a function is just a determination of what theory of reference seems to be the most plausible. It is a metatheoretical function. That is not the kind of function, however, that can help us solve the problems of how the subject is embodied in the world and what the psychologically relevant properties of that embodiment are. What we need is an account of how we know -when we know the meaning of a term or a sentence and when we know how to use it- to what the term or the sentence refers. That kind of knowledge is not to be assimilated to knowing how to tell whether, e.g., 'water' refers to $\mathrm{H}_{2} \mathrm{O}$ or $\mathrm{XYZ}$, i.e., it is not an account of how we might be able to pick out the appropriate referent in all possible contexts. It is an account of how the subject's discriminative abilities get to the referent within a given domain.

Unlike Block's metatheoretical function, such an account relies on our having abilities that depend on our being connected to the objects of our thoughts without the requirement that we have any special conception of those objects. Unlike Block's notion of conceptual role, which is essentially inferential, this account relies primarily on nonconceptual aspects of cognition. Given those two basic differences, Block's claim concerning how the two components in dual factor theories are related is of no help in solving the problems posed by McGinn's contention that they are independent. 


\section{Choice of Dressings.}

Suppose my first aim has been achieved, that I have shown that a proper theory of content must make it possible to establish an internal connection between cognitive role and reference. That would be enough to cast serious doubts on the plausibility of any dual factor theory. But, just in case the reader is not convinced yet of the implausibility of those theories, I now examine the problem from a different perspective, that of the analysis of a type of theory designed, within the dual factor proposal, to deal with the causal explanatory aspect of content: Conceptual Role Semantics.

Conceptual Role Semantics (henceforth CRS) is advertised by Ned Block as the magic recipe that will fulfill the naturalistic dreams of the most demanding semantic gourmet. The reason, he claims, is that this recipe contains as its main ingredient an appropriate notion of content for Psychology. With that notion in hand, all eight conditions that, according to Block, must be met by an acceptable semantics for Psychology can be provided for. Those conditions or desiderata are: (1) explain the relation between meaning and reference/truth; (2) explain what makes meaningful expressions meaningful; (3) explain the relativity of meaning to representational system; (4) explain compositionality; (5) fit in with an account of the relation between meaning and mind/brain; (6) illuminate the relation between autonomous and inherited meaning; (7) explain the connections between knowing, learning, and using an expression, and the expression's meaning; (8) explain why different aspects of meaning are relevant in different ways to the determination of reference and to psychological explanation (Cfr. Block, 1986).

Two possible responses come immediately to mind. One is to question the very desiderata proposed, to argue, e.g., that a proper semantics for Psychology needn't meet those conditions, that there are too many or too few, or just that a proper semantics for Psychology must meet different conditions. The second response is to take the eight conditions for granted, but then to check the advertisement with a consumer's eye, i.e., check whether or not conceptual role semantics can do what has been promised in relation to those conditions.

I pursue this second response and ignore the first. I take for granted, then, that the eight ingredients of Block's proposal are correct in number and content. I discuss 
only whether or not some version of CRS can meet one of the eight nominated conditions: that the theory must explain compositionality (desideratum 4$)^{5}$.

I argue that desideratum 4, that the theory be compositional, cannot be satisfied under any version of CRS. The same conclusion is reached by Fodor and Lepore (Fodor and Lepore, 1992). But their argument is partially undermined by a failure to attend to two possible senses of compositionality. In what follows, I distinguish these two senses and show that although CRS does not provide an account of the more complex compositionality associated with higher level thought, it is, pace Fodor and Lepore, compatible with an alternative kind of compositionality appropriate to a more primitive notion of content.

My argument has three stages. In the first, I make clear that the problem of explaining compositionality is for CRS the problem of individuating conceptual roles. I compare this problem with that of finding a robust notion of content identity in, e. g., Churchland's state space proposal and show that the holistic implications of both accounts have the same origin, namely the lack of identity criteria for constituent tokens of the complex expressions in which they figure. I then describe a possible solution to this problem for CRS that seems to work in the connectionist field. The solution is the notion of functional compositionality as developed by Tim van Gelder (van Gelder, 1990).

In the second stage, I argue for the truth of the following conditional. If we make the causal parameters that enter into the inferential roles of CRS correspond to sensory properties, we can at least individuate very primitive concepts as contentful functions of those parameters.

In the third and final part of my argument, I show that this lower-level notion of compositionality does not suffice for higher order representations. For those concepts that involve natural language, we need a higher-level, concatenative kind of compositionality that cannot be developed out of the theoretical resources of CRS. I conclude with Fodor and Lepore (Fodor and Lepore, 1992) that whatever its other merits, CRS does not meet the fourth of the eight desiderata of Block's recipe.

5 Note that if my previous arguments are correct, I have also shown that desideratum (1), i.e., explain the relation between meaning and reference/truth cannot be satisfied. 


\section{Compositionality versus Compositionality.}

Representationalism is the thesis that, for a system to exhibit any reasonably complex cognitive function or behavior, it must be capable of containing certain structured mental representations. The necessary property that the inner structure of mental representations must have to be useful for explaining cognitive behavior, especially behavior involving systematicity and productivity in thoughts and sentences, is called compositionality. The inner structure of mental representations has thus to be of a compositional kind. Satisfying the condition of being useful for explaining compositionality is, understandably then, an important desideratum in any theory of mental or linguistic content.

The reason a proposal such as CRS is problematic when used to account for compositionality is well known. The core of the problem is the combination of a notion of content characterized as inferential role -and hence, within CRS, as causal role- with the rejection of the analytic / synthetic distinction; a rejection that one must make if one wants to maintain the interest and idiosyncrasy of CRS (Cfr. Fodor \& Lepore, 1992) .

The key to Fodor and Lepore's diagnosis lies in what it is called the principle of isomorphism: that "If a thought / sentence $S$ expresses the proposition that $P$, then syntactic constituents of $S$ expresses the constituents of P" (Fodor \& Lepore, 1992, p. 176). According to Fodor and Lepore, the phenomena of productivity and systematicity can be explained only if mental representations satisfy this principle, i.e., if mental representations have a syntactic inner structure. Compositionality thus means syntactic compositionality. Therefore, if productivity and systematicity are to be explained, the physical instantiations of tokens of the same type have to be identical.

As might be expected, the problem is that conceptual / inferential role is not compositional in this sense. If "conceptual role is total causal role" (Block, 1986, p. 628), there does not seem to be an answer to the question of which inferences or causal relations are constitutive of the meaning of, e.g., the concept DOG and which are not. When the analytic / synthetic distinction is rejected, the meaning of 'dog' is then a function of all the inferences in which it plays any role. And, it is argued, the content of any physical structure identified with the concept-type DOG varies coordinate with the alteration of any systems DOG-involving knowledge. We are thus left with no principle of isomorphism, i.e., no robust notion of content identity onto which to project the syntactic structure required to explain compositionality.

A similar problem afflicts theories that rely on a connectionist account of cognitive processing. The reason is that the connectionist approach likewise does not 
provide tokens that meet the isomorphism constraint. In fact, it has been argued, the main problem for connectionism is the lack of identity criteria for the individuation of contentful states. More precisely, Ramsey, Stich and Garon (1991) and Fodor and Lepore (1992) argue that mental states are not, in the connectionist paradigm, individuable as weight or activation states of the systems. The crucial point is the distributed and superpositional character of representation in connectionist models. The mechanisms responsible for the input / output relationships in these models are the units that form the network. Each unit contributes to encoding information about different representations -that is what superpositional representation means- and each representation is thus distributed across a large set of resources.

As the network's computations are completely determined by activity at the units level, and those units encode at the same time information of many different types, it is not possible to identify a stable and recurrent entity that corresponds to the classical notion of a symbol and that is handled by an independent processing system, i.e., there is nothing like the isomorphic physical identity of tokens of a given concepttype that Fodor and Lepore require.

I use Churchland's state space semantics to illustrate this problem of not being able to produce a robust notion of content identity due to the failure of the principle of isomorphism. I do not discuss Churchland's views in detail. I discuss them only to show that the individuation problems and their far reaching holistic implications both in CRS and in state space semantics have the same root, i.e., the lack of identity criteria for constituent tokens of the complex expressions in which they appear. That is no surprise because both Churchland and CRS reductionist theorists seek to underpin meanings in very similar terms, as mental processes in one case and as causal properties of mental processes in the other. What, at a higher level of description, has a certain unitary meaning, may have, at the level of these reductive bases, a quite complicated and disjunctive individuation scheme.

With this parallelism in mind, I deal with the main aim in this section of the paper, namely, to provide for CRS a solution concerning compositionality that works at least for some connectionist systems. The solution is the notion of functional compositionality developed by Tim van Gelder who contrasts it with the more classical, Fodorian notion of concatenative compositionality. Functional compositionality is -I argue- a suitable kind of compositionality for CRS. To show why, I first rehearse the main features of both notions.

A compositional scheme is concatenative if in generating compound representations out of primitive constituents and in determining the primitive 
constituents of complex expressions, these constituents remain unaltered. Natural languages, logic, and the languages of mathematics and classical AI are concatenative in this way. All of them have a syntactic internal structure, i.e., an internal structure "such that the abstract constituency relations among expression types find direct, concrete instantiation in the physical structure of the corresponding tokens" (van Gelder, 1990, p. 361).

In the contrasting functional kind of compositionality, there are general, effective, and reliable processes for generating complex expressions given their constituents and vice versa, i.e., for determining the primitive constituents of complex expressions. Yet, the complex expressions do not contain their constituents in any kind of familiar syntactic way. There is a significant formal internal structure but it is not a strictly syntactic one. The identity criteria for constituent tokens are not independent of the processes that generate and decompose expressions, i.e., these constituents are not "proper parts" of the complex expressions in which they figure. However, the causal properties of the complex representations as a whole correspond to the causal properties of these constituents.

van Gelder offers a powerful and intuitive example: the Gödel numbering scheme for a formal language. In Gödelese it is always possible, i.e., there are general, effective, and reliable processes, to generate complex expressions given their constituent parts and vice versa. Yet "the Gödel numeral for a complex expression does not literally contain the Gödel numerals of its constituents" (van Gelder, 1990, p. 362). For example, the Gödel number of

$((\mathrm{P} \& \mathrm{Q})$ \& $\mathrm{R})$ is 388282251281722597800000 ;

the one for $(\mathrm{P} \& \mathrm{Q})$ is 51342984000 ;

and the one for $\mathrm{P}$ is 32 .

Yet neither the numeral for $\mathrm{P}$ nor the numeral for $(\mathrm{P} \& \mathrm{Q})$ is present in the numeral for $((\mathrm{P} \& \mathrm{Q}) \& \mathrm{R})$.

End of the rehearsal.

The main problem with the individuation of content in terms of conceptual / causal roles is, according to Fodor and Lepore, the lack of the kind of compositional inner structure that mental representations must have if we are going to be able to explain facts about systematicity and productivity. But this problem loses part of its urgency if we think of the inferential relations posed by the conceptual role theorist as functionally composed entities and thus break the equivalence between Representationalism and the Language of Thought Hypothesis. After all, what is 
relevant for the individuation of content in CRS are causal roles, and an outstanding feature of functional compositionality is precisely that it provides a reliable way of keeping track of the causal properties of the constituents of complex expressions when they figure in those expressions.

As shown above, once you defend representationalism you are committed to compositionality, but that does not involve any constraints on the kind of compositionality that mental representations must exhibit. Once you defend representationalism, you are committed to compositionality, but not necessarily to the Fodorian concatenative compositionality of the LOTH. A further argument is needed if one wants to keep defending that commitment. Such an argument must show that there is no way of building up the features that characterize compositionality within systems that are not designed according to the classical account of cognitive processing. Given van Gelder's notion of functional compositionality and the existence proof of connectionist systems (Cfr. Chalmers, 1990), the construction of such a further argument seems problematic, to say the least.

It is also true that over-the-top optimism concerning connectionist solutions to deep problems in the theory of meaning is generally misplaced and I refer to some of those problems later on. But for now, I remain optimistic and proceed to work out in detail this functional solution to the problem of compositionality within CRS.

\section{How to Fill a Conceptual Role.}

To figure out how this solution might work, I take the parameters, a function of which we determine the inferential / causal role of some primitive concepts in CRS, to be sensory parameters.

Unlike Paul Churchland's, my proposal is to make the dimensions of semantic space correspond to observable / sensory properties. The contents of primitive concepts would then be characterized as functions of those parameters or, if you prefer, as certain patterns of activity in a connectionist hyperspace whose dimensions are sensory properties.

Fodor and Lepore agree that this empiricist ingredient provides a robust identity criterion for state space semantics (Cfr. Fodor and Lepore, 1992, p. 205). If the semantic spaces are identified in CRS with the causal parameters of primitive concepts, then this empiricist ingredient also provides a robust identity criterion for CRS. 
By 'primitive concepts', I mean those concepts knowledge of which is embedded in an efficiently functioning procedure, activated in response to external, sensory stimuli, but not available as knowledge to any other part of the system and therefore not verbally stateable. That is precisely the definition of the first stage of development described in A. Karmiloff-Smith's Representational Redescription Hypothesis (henceforth RRH) (Cfr. Karmiloff-Smith, 1987 and also Clark \& Karmiloff-Smith, 1993) .

The Representational Redescription Hypothesis is a theory meant to explain how knowledge that is initially merely implicit in functioning procedures can be made progressively more explicit and, as an ultimate side-effect of such a process, consciously available. The transition involves the repeated redescription of stored information in new representational formats.

In more detail, Karmiloff-Smith establishes an array of three levels of redescription. The first level is the one just described. Here the knowledge is embedded in special-purpose procedures that are part of the goal of successful negotiation of a very specific domain. The first level representations are domain specific in the simple sense that they are routines that function only in the type of context in which they were initially developed, i.e., they cannot be generalized to other cases where, intuitively, the same knowledge could be fruitfully applied ${ }^{6}$. When this knowledge is available to the system as data, the second level of redescription is possible. This knowledge is then explicitly represented, but not yet consciously accessible and therefore not verbally stateable. According to Karmiloff-Smith, the third (upper) level of redescription coincides with the conscious level and then the knowledge becomes verbally stateable.

The basic idea behind this view is to treat the successful negotiation of a domain as the fundamental notion of a theory of concept acquisition. The endogenous tendency to redescribe this procedural knowledge then makes these representations increasingly independent from specific behavioral contexts. The RRH, and especially the empirical support that it provides in relation to the first stage of development, sheds some light on the idea of primitive concepts as concepts for which CRS can account in terms of lower-level, functional compositionality. The reason is that the components of the representational content that CRS has to explain are here taken to be sensory parameters. CRS can thus provide the means for anchoring semantic primitives through learning.

$6 \quad$ A good example is novice piano playing where the context for the production of the middle bars is set by the playing of the preceding notes, so you cannot begin in the middle. For a more detailed discussion of this limitation see Karmiloff-Smith, 1987 and Clark and Karmiloff-Smith, 1993. 
Furthermore, because in this version of CRS the mastery of the abilities that are equivalent to the individuation of primitive concepts is directly related to features of the experience, that individuation is not circular because it does not have to be explained in terms that presuppose the availability of those concepts. The taxonomy is one of mental states in terms of the causal relations holding among those sensory properties. One can then say that two tokens are tokens of the same concept-type -at least for systems with the same sensory transducers- if they occupy the same causal role in the network of abilities that justify the ascription of that concept. Consider the following example:

We are all familiar with the image of infants' tracking moving objects, stopping moving objects, and reaching for objects of different shapes and weights (Rutkowska, 1993). We could try to account for that kind of behavior by reference to purely internal states of representation and computation. But in doing so, Rutkowska claims, we would miss one of the main components of the perceptual process, namely, the behavioral component.

"[Descriptions of objects and their properties] can be viewed more pragmatically in terms of action programs: virtual mechanisms whose operation selectively exploits task-relevant aspects of multiple descriptions ... to support the direct invocation of behavioral procedures ... Making explicit an aspect of the physical world, such as a surface, over many situations does not entail any ability to represent it as a property that is common to that range of situations, let alone potentially applicable to others" (Rutkowska, 1993, p. 971).

If we opt for this second, action-guided, approach, we must acknowledge that those visually based representations of surfaces cannot be conceptualized independently of behavioral processes. The reason is that those processes can change the viewer's relationship to her or his environment, which in turn changes the information available to visual processing. As those diachronic processes of change are the outcome of natural sensory-motor systems' local interactions with the environment, they can be explained only in terms of a computational account of action. This computational account of action could not be developed without taking the cognizer to be an embedded system whose internal processes are controlled by behavioral interaction with its environment.

Of course, the fact that we can use the concept SURFACE to describe the behavior of infants does not entail that that concept itself "is somehow a component of 
the experience's representational content, nor that the concept must be possessed by the experiencer" (Peacocke, 1992, p. 111). That is, it does not entail that children have the concept SURFACE in any way that can be assimilated to the possession of a syntactic item and that can be governed by a concatenative mode of compositionality à la Fodor. If this was the case, infants could use that concept in various and different domains with flexibility and accuracy, and they cannot.

What children acquire is rather some more primitive concepts in terms of sensory properties that can function in an structured way to accomplish the different movements involving surfaces. The ascription of that primitive concept is also warranted by the mastery of the abilities directed towards handling the observable parameters of that specific domain. This non-epistemic but rather pragmatic notion of conceptual role provides a kind of molecularism for CRS. A pragmatic distinction is used to distinguish between those sensory properties of the conceptual role that are constitutive of a concept's identity and those that are not. This counters the Fodorian move about holism and compositionality in CRS. Or does it?

\section{Going higher.}

Well, not really. There are still some important questions to answer. One of them, probably the most urgent one facing this proposal, is what happens to the rest of our concepts, i.e., what happens when we go higher in the order of representations? Unfortunately, the answer to that question is that, at higher than sensory-based representations, the functional kind of compositionality cannot be sustained.

When the knowledge implicit in the functioning procedures of the primitive concepts becomes explicitly represented and verbally stateable, i.e., when we reach those concepts to which semantic distinctions such as the analytic / synthetic apply, we have to fall back on the higher-level, concatenative kind of compositionality. And the problem lies in the lack of resources in CRS to account for that kind of compositionality.

I do not address this problem here. Fodor and Lepore dwell on the reasons why CRS and concatenative compositionality are not reconcilable. But I say a bit more about why we have to revert to that kind of compositionality. These reasons have already been put forward in a rather different context by those interested in developmental Psychology and Connectionism. In fact, the premises of the Representational Redescription Hypothesis that I rehearsed above make them rather predictable. 
What characterizes this Hypothesis is the view of human development as a progressive redescription of the basic knowledge implicit in domain-specific procedures. The linguistic stage of this development entails the wide, flexible use of this information in possibly very different kinds of domains. At this stage the structure of the representations in relation to linguistic behavior is very evident. The examples are famous. If one can think / say 'Mary likes strawberry cakes' and 'Linda loves cheese cakes', one must also be able to entertain thoughts and / or utter sentences such as 'Linda likes strawberry cakes', 'Mary loves cheese cakes', or 'Linda loves Mary'.

The concepts involved in these examples are too far from the empiricist frame to allow the limits of their inner structure to be defined in terms of functional compositionality along sensory axes. In going higher from the primitive, sensory-based concepts, to the concepts involved in linguistic behavior, we face typical problems such as those of context sensitivity and collateral information. To solve these problems we must devise a symbolic structure over which we can define direct operations in the form of rules that are context-free.

The case is again parallel to that of connectionist systems. One way of approaching the problem of designing connectionist models in which the behavior to be modeled involves representations of higher order than the ones based on observable properties is precisely to reduce the level of distribution of the representations by defining the symbolic structures directly into the hardware, into the architecture of the network, or by other methods. One way or another, the bet seems to be for some sort of hybrid models in which the lowest level procedures are broadly connectionist but the higher level redescriptions are broadly symbolic (Cfr. Hendler, 1989).

What CRS needs, then, is some way of grouping the partitions of sensory causal relations that constitute the primitive concepts into more coarse-grained constituents onto which to project proper semantic categories. To be able to do that, i.e., to be able to cluster together different micro-primitive-concepts into macro-higher-order-concepts, we need some previous semantic criteria -probably with a strong normative characterthat allows us discriminate between which micro-constituents belong to which macroconcepts and which do not.

But those semantic criteria cannot be easily built out of the CRS theoretical resources. For one thing, its reductionist character makes it difficult to incorporate normative criteria at all. Besides that, it does not seem easy to build a principled 
distinction that lets us discriminate without circularity between those inferences that will count as part of the meaning of a concept and those that will not ${ }^{7}$.

From an empirical point of view, this problem of grouping criteria can be solved in more or less elegant ways. To implement symbolic structures directly in the network architecture is one solution in connectionism. The RRH is one solution in Psychology. But, from the philosophical point of view of CRS, a proper account of the relations between micro-conceptual constituents and macro-conceptual-concepts is still needed and until that account is provided, as a candidate for a proper semantic approach, CRS still faces problems.

We might therefore apply to CRS a comment originally made regarding Churchland's proposal, i.e., that it is not a semantic theory, but rather a theory of mental processes (Cfr. Fodor \& Lepore, 1992, pp. 200-201). The reductionist character of CRS seems to justify that diagnosis. That would also explain the suitability of functional compositionality within both connectionism and CRS at the level of primitive representations and why that kind of compositionality does not provide for the kind of systematicity / productivity phenomena, at the semantic level of propositional knowledge, that Fodor and Lepore are thinking of. If that is true, then CRS cannot be an appropriate semantics for Psychology in the sense that Block and other dual factor theorists intended it to be. At any rate, even if that diagnosis is false, it should be clear after what I have said here that the kind of compositionality that a proper semantic theory requires cannot be easily provided by CRS. Therefore, functional compositionality notwithstanding, something is still missing from inside the conceptual role.

\section{Bridging Tricks. Conclusion.}

McGinn's case for the theoretical independence of the causal explanatory aspect of content and its referential truth-conditions rests on the dual aspect of internal representation. Against this, I argue, in the first part of this paper, that the requirement of establishing a difference between those two aspects of representations does not in

$7 \quad$ As a semantic proposal, CRS theorists should aim at establishing, in an informative and noncircular way, in what consists the knowledge of the meaning of the expressions of a language that the speakers of that language have. Now, inferential processes are inherent in the meaning of certain expressions and sentences. So, whatever counts as establishing the knowledge of the meaning of those expressions cannot be -if we want to avoid circularity- the very same inferential processes -deductive behavior- that, according to CRS, define that meaning. 
itself provide any justification for positing the orthogonality of the theories concerned with that dual structure. Furthermore, I show that the split status of dual factor theories unfits them for the explanation of how it is possible for the cognitive subject's thoughts to become world-engaged or, to be more precise, to become successfully world-engaged. If the arguments in Section 2 are correct, then, convenient though it may be, we have no reason to believe that a theory concerned with (the duplex structure of) content ought to be cashed out in the way dual factor theorists intend it to be.

In the second part of the paper (Sections 3-6) I follow a different strategy. There my aim is to show that, when focusing on the plausibility of the dual factor project from the point of view of one of the theories involved in it, namely, Conceptual Role Semantics, the outcome is equally negative. Through a three stage argument, and choosing compositionality as the key desideratum to be satisfied by any version of CRS, I show that, although there is a notion of compositionality (functional compositionality) that can be accounted for within CRS, it works only for representations that do not involve language. When going higher, i.e., when reaching the linguistic level of representations, CRS cannot cope with the requisite kind of compositionality (concatenative compositionality). Again, if my argument is correct, then any dual factor theory that incorporates CRS as the theory concerned with the causal explanatory aspect of content is doomed.

The conclusion to be drawn at this point is the following: either dual factor theories fail to be an appropriate approach to content due precisely to its -independentdual character or they fail due to the constitutive lack of resources to deal with the concatenative compositionality of language-involving representations. Either way, we can construct a case that weighs heavily against such theories. My primary lesson conclusion is thus that we must confront the issue of the content of mental and linguistic representations in terms that do justice to both its dimensions. This implies doing justice to the bridge that links them. Twin pleas for a theory of content. 


\section{References}

Block, N. 1986: "Advertisement for a Semantics for Psychology," in P. French, T. Uehling and H. Wettstein, eds., Midwest Studies in Philosophy, vol. 10: Studies in the Philosophy of Mind. Minneapolis: University of Minnesota Press, pp. 615-678.

Chalmers, D. 1990: "Syntactic Transformations on Distributed Representations." Connection Science 2, pp. 53-62.

Clark, A. 1994: Associative Engines. Connectionism, Concepts and Representational Change. Cambridge, Massachusetts: MIT Press.

Clark, A. \& Karmiloff-Smith 1993: "The Cognizer's Innards." Mind and Language 8, (4), pp. 487-519.

Dummett, M. 1981: The Interpretation of Frege's Philosophy. London: Duckworth.

Field, H. 1977: "Logic, Meaning and Conceptual Role." The Journal of Philosophy 74 (7), pp. 379-409.

Fodor, J. and Lepore, E. 1992: Holism. A Shopper's Guide. Oxford: Blackwell.

Frege, G. 1892: "Über Sinn und Bedeutung." Zeitschrift für Philosophie und philosophische Kritik NF 100. Trans. in M. Black and P. Geach, eds., 1952 Translations from the Philosophical Writings of Gottlob Frege. Oxford: Basil Blackwell.

Hendler, J. A., ed. 1989: Special Issue: Hybrid Systems (Symbolic / Connectionist). Connection Science 1, (3).

Karmiloff-Smith, A. 1987: "Beyond Modularity: A Developmental Perspective on Human Consciousness." Transcript of invited talk given to the Annual Meeting of the British Psychological Society, April, 1987.

Lewis, D. 1979: "Attitudes de dicto and de se." Philosophical Review 88, pp. 513-549.

McGinn, C. 1982: "The Structure of Content," in A. Woodfield, ed., Thought and Object. Oxford: Clarendon Press, pp. 207-258.

McGinn, C. 1989: Mental Content. Oxford: Blackwell.

Peacocke, C. 1992: "Scenarios, Concepts and Perception," in T. Crane, ed., The Contents of Experience. Essay on Perception. Cambridge: Cambridge University Press, pp. 105135.

Ramsey, W., Stich, S. \& Garon, J. 1991: "Connectionism, Eliminativism and the Future of Folk Psychology," in W. Ramsey, S. Stich and D. Rummelhart, eds., Philosophy of Connectionist Theory. Hillsdale, NJ: Lawrence Erlbaum, pp. 199-228. 
Rutkowska, J. C. 1993: "Ontogenetic Constraints on Scaling-Up Sensory-Motor Systems", Proceedings of the Second European Conference on Artificial Life, vol. 2. Brussels, May, 24-26, pp. 970-979.

van Gelder, T. 1990: "Compositionality: A Connectionist Variation on a Classical Theme." Cognitive Science 14, pp. 355-384.

Wright, C. 1986: Realism, Meaning and Truth, Oxford: Blackwell. 